\title{
A central review of histopathology reports after breast cancer neoadjuvant chemotherapy in the neo-tango trial
}

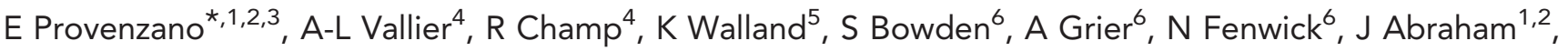
M Iddawela ${ }^{1,2,7,8}$, C Caldas ${ }^{1,2,7}$, L Hiller ${ }^{9}$, J Dunn ${ }^{9}$ and H M Earl ${ }^{1,2}$

${ }^{1}$ Department of Oncology Box 193, University of Cambridge, Addenbrooke's Hospital, Hills Road, Cambridge CB2 00Q, UK; ${ }^{2}$ Cambridge Experimental Cancer Medicine Centre and NIHR Cambridge Biomedical Research Centre Box 277, Hills Road, Cambridge CB2 0QQ, UK; ${ }^{3}$ Department of Pathology, University of Melbourne, Grattan St, Parkville, Victoria, Australia; ${ }^{4}$ Department of Oncology, Cambridge Clinical Trials Centre, Box 279 (S4) Addenbrooke's Hospital, Cambridge CB2 0QQ, UK; ${ }^{5}$ School of Clinical Medicine, Addenbrooke's Hospital, Hills Road, Cambridge CB2 0QQ, UK; ${ }^{6}$ Cancer Research UK Clinical Trials Unit, Institute for Cancer Studies, University of Birmingham, Edgbaston, Birmingham, B15 2TT, UK; ${ }^{7}$ Cancer Research UK Cambridge Research Institute, Cambridge, UK; ${ }^{8}$ Consultant Medical Oncologist \& Senior Lecturer, Rural Clinical School, University of Melbourne, Shepparton, Victoria, Australia, 3630 and ${ }^{9}$ Warwick Clinical Trials Unit, University of Warwick, Gibbet Hill Road, Coventry CV4 7AL, UK

Background: Neo-tAnGo, a National Cancer Research Network (NCRN) multicentre randomised neoadjuvant chemotherapy trial in early breast cancer, enroled 831 patients in the United Kingdom. We report a central review of post-chemotherapy histopathology reports on the surgical specimens, to assess the presence and degree of response.

Methods: A central independent two-reader review (EP and HME) of histopathology reports from post-treatment surgical specimens was performed. The quality and completeness of pathology reporting across all centres was assessed. The reviews included pathological response to chemotherapy (pathological complete response (pCR); minimal residual disease (MRD); and lesser degrees of response), laterality, the number of axillary metastases and axillary nodes, and the type of surgery. A consensus was reached after discussion.

Results: In all, 825 surgical reports from 816 patients were available for review. Out of 4125 data items there were 347 discrepant results (8.4\% of classifications), which involved 281 patients. These involved grading of breast response (169 but only 9 involving pCR vs MRD); laterality (6); presence of axillary metastasis (35); lymph node counts (108); and type of axillary surgery (29). Excluding cases with $\mathrm{pCR}$, only $45 \%$ of reports included any comment regarding response in the breast and $30 \%$ in the axillary lymph nodes.

Conclusion: We found considerable variability in the completeness of reporting of surgical specimens within this national neoadjuvant breast cancer trial. This highlights the need for consensus guidelines among trial groups on histopathology reporting, and the participation of histopathologists throughout the development and analysis of neoadjuvant trials.

\footnotetext{
*Correspondence: Dr E Provenzano; E-mail: elena.provenzano@focuspathology.com.au

Previous presentations of the study: Oral presentation of preliminary results at the British Breast Group Winter Scientific Meeting, 30 January 2009 Poster presentation of results: early breast cancer (EBC): First report of the primary endpoint - pathological complete response (pCR). (ASCO 2009. Manuscript 3D US assessment: (Gounaris et al, 2011). Manuscript including patients from Neo-tAnGo(lsmail et al, 2010).
}

Received 6 August 2012; revised 15 November 2012; accepted 16 November 2012; published online 8 January 2013

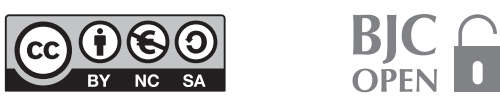


Neoadjuvant chemotherapy is being used increasingly in the management of breast cancer, and has been shown to be as efficacious as adjuvant therapy (Rastogi et al, 2008). There are established clinical indications for neoadjuvant treatment such as locally advanced disease including inflammatory breast cancer (Dawood et al, 2011), and downstaging of large tumours to allow breast conserving surgery rather than mastectomy (Bonadonna et al, 1990; Wolmark et al, 2001). Another benefit of neoadjuvant therapy is in the context of clinical trials, where it offers a unique opportunity for the evaluation of treatment response with pCR acting as a surrogate marker of survival. This allows more rapid assessment of the efficacy of new chemotherapeutic agents, also enabling early cessation of ineffective treatments and providing an opportunity to individualise patient treatment at an early stage. In addition, tumour samples can be collected before, during and after treatment enabling translational research to identify markers of response so target patient populations can be identified (Berruti et al, 2011).

Clinical trials need to recruit large numbers of patients to achieve sufficient statistical power to detect a treatment effect. The most efficient way to do this is via multicentre studies, allowing suitable subjects to be drawn from a much larger patient population. One major disadvantage of multicentre trials in the neoadjuvant setting is that the post-treatment excisions are reported by a variety of pathology departments, with large variation in specimen handling protocols and reporting styles. At present, there are no established national guidelines in the United Kingdom for the histopathological handling of surgical tissue from neoadjuvant cases, and the reporting of neoadjuvant chemotherapy breast specimens. In the literature, there are several different systems for the histological grading of chemotherapy response (Marchio and Sapino, 2011). Many of the suggested systems in common use, rely on a comparison between tumour cellularity of the pre-treatment core biopsy and the post-treatment resection specimen (Figure 1), with the grade of response determined by the reduction in overall cellularity (Chevallier et al, 1993; Sataloff et al, 1995; Smith et al, 2002; Ogston et al, 2003; Pinder et al, 2007). The MD Anderson group have proposed an alternative system, the Residual Cancer Burden (RCB), that derives a score based on tumour bed volume, average tumour cellularity, number of involved lymph nodes and the size of the largest metastasis (Symmans et al, 2007). They have shown a relationship between RCB and survival outcome. The score relies on absolute cellularity post-treatment, without considering the change from pre-treatment cellularity. Traditional tumour grading systems used in the adjuvant setting, including the Nottingham Prognostic Index (NPI) and the TNM system using the prefix ' $y$ ' to indicate neoadjuvant treatment, have also been shown to retain their prognostic value post-chemotherapy (Chollet et al, 2003; Carey et al, 2005).
In the setting of neoadjuvant clinical trials, histopathologists are a vital part of the trial as thorough specimen handling and accurate histological reporting is essential to correctly assess the primary end point (Sahoo and Lester, 2009). In many multicentre neoadjuvant chemotherapy trials to date, patient outcome is established by reporting of pathology data from the treating hospital without any (minimal) guidelines for specimen handling or centralised review of histopathology reports and/or slides to ensure validity and reproducibility of results. The Neo-tAnGo trial was a multicentre phase III randomised study examining the addition of gemcitabine to a standard neoadjuvant therapy regimen of epirubicin and cyclophosphamide with paclitaxel. The primary end point was pCR in both the breast and axilla. A central review of the post-treatment histopathology reports within the Neo-tAnGo trial was carried out, not only to assess the presence of chemotherapy response but also to examine variation in the quality and completeness of pathology reporting between centres.

\section{MATERIALS AND METHODS}

The Neo-tAnGo trial was a multicentre phase III randomised study of 831 patients from 57 centres examining the addition of gemcitabine to a standard neoadjuvant therapy regimen of epirubicin and cyclophosphamide with paclitaxel. The primary end point is pCR in the breast and axilla. Patient eligibility, treatment protocols and preliminary results have been described previously (Earl et al, 2009).

Histopathology reports were requested for all trial patients from 92 centres as per the trial protocol. The reports were received by the trial co-ordinating centre and were anonymised with respect to all patient details, with only the neo-tAnGo trial number available to reviewers. The reports were then independently reviewed by two study investigators, the study histopathologist (EP) and the chief investigator of the study, a medical oncologist (HME). A central pathology review form was completed by each reviewer, collecting information on the laterality of the surgery; whether or not there had been a pCR in the breast (yes/no/unknown); the absence of metastatic disease in the axillary lymph nodes post-treatment (yes/no/unknown); the nature of axillary lymph node surgery (pre-treatment sentinel lymph node biopsy (SLN), post-treatment SLN and/or post-treatment axillary clearance (ALND)) and the number of lymph nodes sampled and the number of lymph nodes involved for each type of surgery. In cases where there had not been a PCR in the breast, the reports were further subcategorised as minimal residual disease $(>90 \%$ response), chemotherapy response present, no chemotherapy response and no comment regarding chemotherapy response. A subset of cases was also independently reviewed by a third party (KW). Following collation
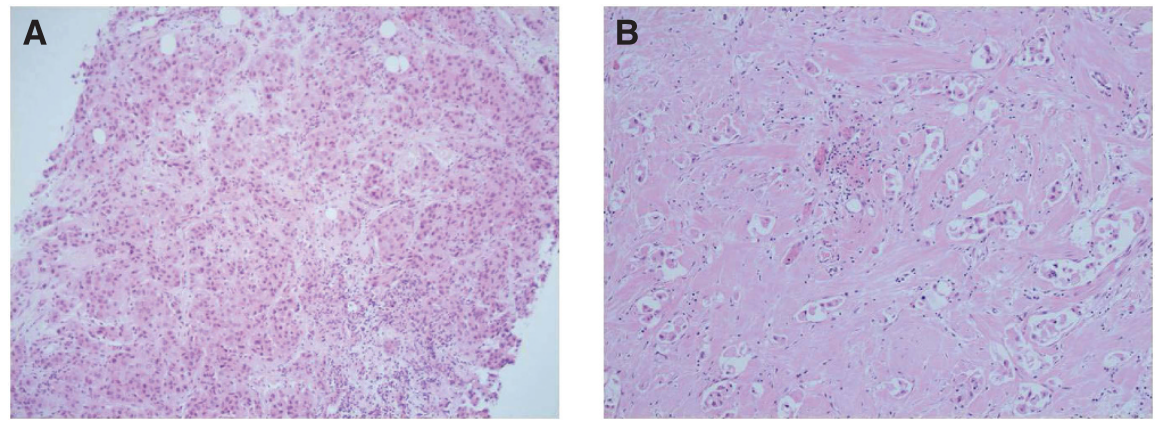

Figure 1. (A) Pre-treatment core biopsy and (B) post-chemotherapy excision specimen showing good response to neoadjuvant chemotherapy with a marked reduction in cellularity following treatment. 


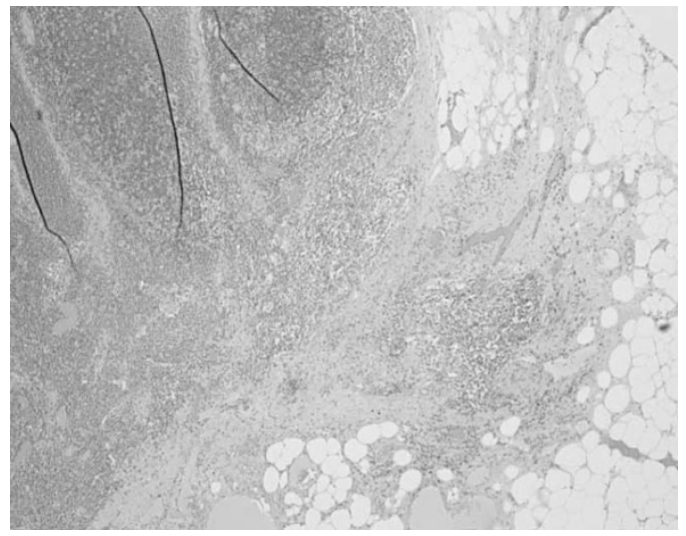

Figure 2. Lymph node showing metastasis with response to treatment with a focal area of fibrosis containing haemosiderin laden macrophages.

of the review findings, cases in which there was disagreement between reviewers were discussed jointly and a consensus was reached. Further information was recorded by one of the reviewers (EP) regarding the number of histology blocks taken in cases with a $\mathrm{pCR}$, whether there was formal grading of chemotherapy response and what system was used, and whether or not the reports commented on the presence or absence of changes suggesting response to chemotherapy in the axillary lymph nodes (Figure 2).

\section{RESULTS}

Histopathology reports were received for review from 816 of 831 patients. Ten patients had bilateral disease, making a total of 826 breast reports reviewed. One patient refused surgery and only had a core biopsy after treatment, this case is excluded from further analysis although of note it was classified as PCR by one reviewer and breast response unknown by the other.

The review of the reports looked at five main pieces of data; laterality, breast response, the presence of axillary metastases, axillary node number and type of axillary node surgery. For the 825 breast operations this gives a total of 4125 data points. There were 347 discrepancies between the two reviewers involving 281 subjects, representing $8.4 \%$ of classifications. Forty-eight subjects had two discrepancies, eight subjects had three discrepancies and one subject with bilateral disease had four discrepancies. The discrepancies were in the following areas; 6 - laterality, 169 - grading of breast response, 35 - presence of axillary metastasis, 108 - lymph node counts and 29 - type of axillary lymph node surgery.

Interpretation of response in the breast. The 169 discrepancies regarding grading of response in the breast are detailed in Table 1. Nine were pCR vs MRD, and in four of these cases there was no axillary metastasis so this potentially altered the primary end point for these subjects. The commonest discrepancies were chemotherapy response vs no comment (82) and MRD vs chemotherapy response (45). The oncologist and chief investigator (HME) tended to interpret the pathology report as indicating the presence of chemotherapy response more frequently than the pathologist (Figure 3). This was because the oncologist interpreted any mention of necrosis or fibrosis as indicating chemotherapy response, whereas the pathologist required an explicit statement within the report as to the presence of chemotherapy response, since both necrosis and fibrosis are commonly seen in breast cancer histopathology in the absence of chemotherapy.
Table 1. Reviewer discrepancies with chemotherapy response status

\begin{tabular}{|l|c|c|}
\hline $\begin{array}{l}\text { Change to final } \\
\text { response } \\
\text { status }\end{array}$ & $\begin{array}{c}\text { Reviewer 1 agrees } \\
\text { with final response } \\
\text { status }\end{array}$ & $\begin{array}{c}\text { Reviewer 2 agrees } \\
\text { with final response } \\
\text { status }\end{array}$ \\
\hline MRD to pCR & $1(1 \%)$ & $1(1 \%)$ \\
\hline pCR to MRD & $3(2 \%)$ & $4(2 \%)$ \\
\hline CRNG to MRD & $6(4 \%)$ & $1(1 \%)$ \\
\hline MRD to CRNG & $1(1 \%)$ & $37(22 \%)$ \\
\hline CRNG to NC & $9(5 \%)$ & $47(28 \%)$ \\
\hline NC to CRNG & $8(5 \%)$ & $18(11 \%)$ \\
\hline NR to CRNG & $1(1 \%)$ & $3(2 \%)$ \\
\hline MRD to NC & $1(1 \%)$ & $10(6 \%)$ \\
\hline NC to MRD & $3(2 \%)$ & $1(1 \%)$ \\
\hline MRD to NR & & $1(1 \%)$ \\
\hline NC to NR & & $9(5 \%)$ \\
\hline NR to NC & & \\
\hline
\end{tabular}

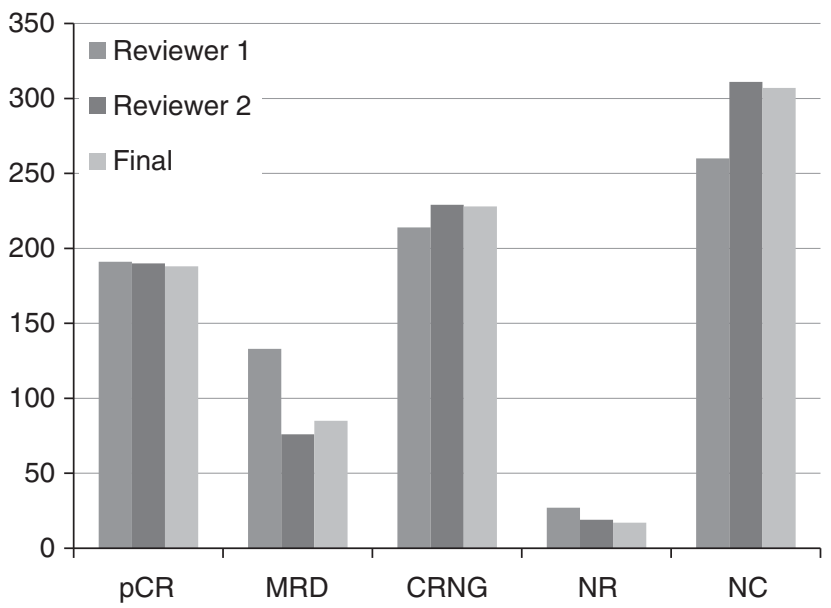

Figure 3. Comparison of scores of chemotherapy response from reviewer 1 and reviewer 2 with final score. ( $\mathrm{PCR}$ - pathological complete response: MRD - minimal residual disease: CRNG chemotherapy response non-gradable: NR - no response: NC - no comment in report on chemotherapy response).

Interpretation of axillary lymph nodes. Of the 35 disagreements regarding the presence of axillary metastasis, 7 were in patients who had a pre-treatment SLN with no further axillary surgery; one of the reviewers called these negative while the other called them 'not known'. Midway through the review it was agreed to call these cases negative for post-treatment metastasis. A further nine discrepancies were errors secondary to the wording of the form; 'Yes' indicating an 'absence of metastasis'. The remaining cases relate to differences in interpretation of the pathology findings such as isolated tumour cells (ITC's) (5), cells identified by immunohistochemistry only (3), deposits in axillary nodal fat (2), scarring in lymph nodes (1) and involvement of an intramammary lymph node (1).

There was disagreement in the number of lymph nodes, including the number of nodes sampled and/or the number of nodes involved, in 108 cases. In 52 cases, the source of the disagreement was not recorded. For the remaining cases, reasons for disagreement included interpretation of ITC's (13), errors/ inconsistencies in the pathology report, for example, different 
Table 2. Classification of chemotherapy response within the breast

\begin{tabular}{|l|c|}
\hline Final grading of breast response & \\
\hline pCR & $188(23 \%)$ \\
MRD & $85(10 \%)$ \\
Chemotherapy response present & $228(28 \%)$ \\
$17(2 \%)$ \\
No response & $307(37 \%)$ \\
No comment re-chemotherapy response & \\
\hline All non-pCR cases & $287(45 \%)$ \\
\hline Formal comment re-chemotherapy response & $350(55 \%)$ \\
\hline No comment re-chemotherapy response
\end{tabular}

numbers recorded in the microscopic findings and conclusion (13), missed pre-treatment SLNs (8), missed delayed ALND (5), nodes positive on IHC only (4), classification of internal mammary and intramammary lymph nodes (3), the presence of multiple specimens (2) and missed axillary tail lymph nodes in mastectomy specimens (2). In 29 cases, the discrepancy was in the type of lymph node surgery; 18 SLN vs ALND, 6 the classification of axillary sampling specimens and 5 pre- $v s$ post-treatment.

Final consensus classification of chemotherapy response. The final classification for the 825 cases based on the combined review of the breast reports was pCR in 188, MRD in 85 , chemotherapy response present in 228, no response in 17 and no comment regarding response in 307 (Table 2). In cases classified as MRD, the presence of chemotherapy response had been mentioned in 45 reports but only 13 of these reports included formal grading of response, with one stating 'MRD', seven giving a percentage response $>90 \%$ and five giving a Miller-Payne score of 4 . The remaining cases were classified as MRD by the reviewers on the basis of invasive tumour size $<5 \mathrm{~mm}$, the presence of DCIS with microinvasion, scattered single cells detectable by immunohistochemistry or the presence of lymphovascular space invasion only. Overall, only 287 of the 637 (45\%) reports from patients who did not achieve pCR contained a specific reference to the presence of chemotherapy effect. Of these, only 55 (8.6\%) gave some sort of formal grading of response, with 20 using the Miller-Payne grading score, 20 giving a percentage change in cellularity, one stating that there was 'MRD' and 14 stating that there was 'no response'.

Nature of axillary surgery and assessment of axillary response. The lymph node procedures performed are summarised in Table 3. In all, 154 out of $816(19 \%)$ patients underwent a SLNB, 60 out of 154 (39\%) before commencement of chemotherapy and 94 out of $154(61 \%)$ after completion of chemotherapy at the time of their breast surgery. Of the 60 patients who had a pre-treatment SLNB, 26 went on to have a post-chemotherapy ALND; 3 of these patients had a negative pre-treatment SLNB. Forty patients who had a posttreatment SLNB went on to an ALND, 29 as an immediate and 11 as a delayed procedure. Thirteen patients with a positive posttreatment SLNB had no further surgery; one had ITC's, two had micromets, two had metastases elsewhere and for the remaining 8 the data are missing. In all, 668 patients (81\%) had an ALND as their only axillary surgery, and 3 patients underwent no axillary surgery.

In total, 788 patients had some form of post-chemotherapy lymph node surgery, and 433 (55\%) of these had axillary metastases. In all, 44 of these patients had a pCR in the breast, and the axillary disease was the only residual tumour present. It was decided to call the presence of any metastatic disease in the axilla positive including isolated tumour cells (ITC's). In all, 36 patients had documented ITC's post-chemotherapy; in 20 (55\%) reports these were regarded as positive, and in the remaining 16
Table 3. Nature of lymph node surgery and nodal status

\begin{tabular}{|l|c|c|c|}
\hline $\begin{array}{l}\text { Lymph node } \\
\text { surgery }\end{array}$ & \multicolumn{1}{|c|}{$\begin{array}{c}\text { No } \\
\text { metastases }\end{array}$} & $\begin{array}{c}\text { Metastases } \\
\text { present }\end{array}$ \\
\hline SLNB - total & 154 & & 23 \\
\hline Pre-chemo & 60 & 37 & 44 \\
\hline Post-chemo & 94 & 50 & 420 \\
\hline ALND - total & 734 & 314 & 379 \\
\hline Only axillary surgery & 668 & 289 & 10 \\
\hline+ Pre-SLNB & 26 & 16 & 31 \\
\hline+ Post-SLNB & 40 & 9 & $115 / 433(27 \%)$ \\
\hline Comment regarding & & $125 / 355(35 \%)$ & \\
\hline Nodal response & & & \\
\hline
\end{tabular}

(45\%) reports the nodes were called negative. In four of these patients ITC's represented the only nodal disease so the interpretation of ITC's changed the nodal status; however, all of these patients had residual disease in the breast so it did not alter the overall response status. When trying to determine response in the lymph nodes, only 238 out of 788 (30\%) reports made a specific comment regarding the presence or absence of chemotherapy related changes; 125 out of 355 (35\%) reports with negative lymph nodes and 115 out of 433 (27\%) reports with positive lymph nodes.

Review of specimen handling. To look at the degree of specimen sampling in patients with a pCR, the pathology reports were examined to determine the number of blocks taken to search for residual disease. For 91 out of 188 cases (48\%), the block description did not form part of the pathology report and it was impossible to determine the number of blocks taken. In the remaining 98 cases, the number of blocks taken ranged from 5 to 81 , with large blocks regarded as equivalent to 4 small blocks. Twelve cases had less than 10 blocks, 55 cases had 10-19 blocks, 14 cases had 20-29 blocks and for 16 cases 30 or more blocks were taken.

\section{DISCUSSION}

Neoadjuvant chemotherapy is being increasingly accepted as firstline treatment for primary breast cancer. It is the preferred treatment option for locally advanced disease particularly inflammatory breast cancer, and can be used in an attempt to downstage large tumours to allow breast conservation surgery. There is also emerging evidence that it may be beneficial in certain patient subgroups, such as women under the age of 50 and women with ER-negative disease (Rastogi et al, 2008; Berruti et al, 2011). The assessment of tumour response to chemotherapy provides important prognostic information for ongoing patient management, with pCR showing a strong association with improved DFS and OS. However, the degree of response remains a predictor of treatment efficacy even in the absence of $\mathrm{pCR}$, and this may be particularly relevant in tumour subtypes where PCR is uncommon such as ER positive/luminal cancers. In patients that respond poorly or progress on neoadjuvant therapy, outcomes are poor and it provides the opportunity to change chemotherapy regimens or trial novel agents. However, results from the former approach have been disappointing thus far (von Minckwitz et al, 2008) and at present, although information about the amount of residual tumour is prognostically useful, it does not typically alter patient management.

The other advantage of neoadjuvant therapy is in the setting of clinical trials such as the neo-tAnGo trial. pCR represents a surrogate end point for survival in this context, enabling more 
rapid analysis of results and the use of smaller patient cohorts (Generali et al, 2011). The neoadjuvant setting also provides a unique opportunity for translational research, with the ability to collect tumour samples before, during and after treatment to examine the effects of chemotherapy on tumour biology and identify novel markers of response.

An example of this approach is measuring Ki67 labelling index after a short course of neoadjuvant endocrine therapy (Dowsett et al, 2009). A high Ki67 labelling index 2 weeks after commencing treatment is associated with poor response to endocrine therapy, with the option of introducing chemotherapy in these patients.

In the neo-tAnGo trial, pCR was defined as absence of invasive carcinoma in both the breast and axillary lymph nodes, with residual DCIS in the breast permitted. This is now the most widely accepted definition of pCR. Previous studies, such as the NSABP B18 and B27 trials, regarded pCR as absence of residual invasive cancer in the breast only; residual axillary metastatic disease was accepted (Rastogi et al, 2008). In these trials, post-operative nodal status was a strong independent predictor of both DFS and OS. Other studies have also identified axillary pCR as an important marker of improved outcome, even in patients who do not achieve pCR in the breast (Rouzier et al, 2002; Hennessy et al, 2005; Sakakibara et al, 2009). Interestingly in 44 patients in the neo-tAnGo study there was residual axillary nodal disease despite a pCR in the breast. This may represent a true difference in chemosensitivity of metastatic disease $v s$ the primary tumour, and in some cases there is minimal change in axillary nodal deposits despite the presence of marked chemotherapy effect in the primary cancer (EP personal observation). Alternatively, it may in part represent a sampling issue, with axillary lymph nodes being entirely embedded compared with more limited sampling of the primary tumour bed which may be quite extensive or difficult to localise. The other controversy is whether or not to include residual DCIS in pCR. Several studies have now shown that the presence of DCIS does not alter prognosis in patients with complete eradication of the invasive cancer (Jones et al, 2006; Mazouni et al, 2007). In addition, excluding cases with DCIS narrows the pCR group and may confound the results of biomarker studies (Mazouni et al, 2007).

As $\mathrm{pCR}$ is the primary end point in neoadjuvant trials, the histopathologist assumes a pivotal role as thorough specimen handling and accurate reporting of results is vital to correctly assigning patient outcome. Histopathologists should be engaged as key members of the clinical trial team in participating centres, and criteria for specimen handling and reporting should be included within the trial design. This central review of pathology reports in a multicentre trial has revealed huge variation between laboratories. At present there are no national UK consensus guidelines for handling and reporting of neoadjuvant breast chemotherapy specimens, although this will be addressed in the forthcoming updated edition of the UK guidelines (personal communication, Professor SE Pinder).

Ideally, while handling a neoadjuvant chemotherapy specimen, the pathologist should have access to the pre- and post-treatment imaging findings so they know the number of tumour foci and their site and size. The sampling of neoadjuvant specimens will be influenced by whether or not there is a residual macroscopic lesion, the presence of a clip indicating the tumour bed, and the extent of disease on imaging pre-treatment. Recommendations in the literature are often vague, ranging from six or more sections from a mastectomy specimen including at least one section from the nipple (Chevallier et al, 1993), 'multiple' sections from the biopsy site and random sections of the nipple and four quadrants (Sataloff et al, 1995), to a minimum of 10-15 blocks in cases where there is no palpable tumour (Buzdar, 2007). In the Miller-Payne scoring system it is recommended at least four sections be taken if there is macroscopic residual tumour or the whole face of the tumour scar should be sampled, and a patient can only be regarded as having had a grade 5 response ( $\mathrm{pCR}$ ) if the previous tumour site is identified histologically in the form of a fibroelastotic scar (Ogston et al, 2003). The placement of radio-opaque clips in the tumour bed prior to commencement of chemotherapy is important for localisation for breast conserving surgery, and has been shown to reduce local recurrence rates (Oh et al, 2007). However, even in patients undergoing mastectomy, the presence of a marker is an extremely useful aid for the pathologist in accurately localising the site of the tumour when there has been an excellent response with no gross residual disease, resulting in more efficient sampling and reliable determination of the presence of $\mathrm{pCR}$.

In patients who did not achieve pCR in this review, there was a comment regarding the presence of chemotherapy effect in the breast in only $45 \%$ of cases, with formal grading of response in $<10 \%$. Non-pCR cases include a huge spectrum of tumour response, from near eradication of disease to a complete absence of treatment effect. Quantification of residual disease is important, as in patients who do not achieve a pCR the degree of chemotherapy response is still a predictor of treatment efficacy. Studies using both the Miller-Payne scoring system and the Residual Cancer Burden have shown decreasing tumour cellularity correlates with better survival outcomes in the absence of pCR (Ogston et al, 2003; Symmans et al, 2007). Similar outcomes have been shown between patients with pCR and those with MRD (Penault-Llorca et al, 2008). Reporting on the presence of chemotherapy effect in the axillary lymph nodes within neo-tAnGo was even worse, with less than a third of reports commenting on nodal response. This is particularly important in patients who are node negative post-chemotherapy, as clinical pre-treatment nodal status is an important marker of outcome and risk of local recurrence, and the presence of pre-treatment nodal metastases may be used in clinical decision making, particularly with regards to adjuvant radiotherapy (Dawood et al, 2008; Papa et al, 2008). The reasons for the poor reporting of nodal response are uncertain; some systems for grading chemotherapy response, such as the original description of the Miller-Payne system, do not include separate scoring of nodal response, and there may be a lack of understanding of the clinical significance of nodal response by pathologists.

The poor reporting of chemotherapy response other than $\mathrm{pCR}$ in this review highlights the need for consensus guidelines for the handling of neoadjuvant chemotherapy specimens. A formal grading system needs to be included in reports; however, there are several systems in the literature each with advantages and disadvantages (Pinder et al, 2007; Marchio and Sapino, 2011). The only system used by study centres in the neo-tAnGo trial was the Miller-Payne system. This is a relatively easy to use system that divides response into five grades, with grade 1 being no change or alteration in cell morphology with no reduction in cellularity, grade 2 being up to $30 \%$ loss of cellularity, grade $330-90 \%$ loss of cellularity, grade $4>90 \%$ loss of cellularity and grade 5 being fibrous stroma with no identifiable tumour cells but DCIS permitted (pCR) (Ogston et al, 2003). The main disadvantages are that it grades response in the breast only, and requires comparison between cellularity in the pre-treatment core biopsy and the excision specimen. The pre-treatment core biopsy may not be available if it was performed in another centre, and it must be assumed cellularity in the core is representative of tumour cellularity as a whole. Similarly, there is the dilemma of how to report cases where there has been a marked reduction in the size of the tumour but the residual tumour remains highly cellular. An alternative grading system, the Residual Cancer Burden, derives a score based on tumour bed volume, average tumour cellularity, number of involved lymph nodes and the size of the largest metastasis (Symmans et al, 2007). The score relies on absolute cellularity post-treatment, which has the advantage of not requiring the pre-treatment core biopsy to grade response. However, it is relatively labour intensive as cellularity must be assessed on several slides, and tumour size must be accurately 
measured in two dimensions, which can be difficult post-chemotherapy when there is no residual macroscopic lesion. It cannot be applied in patients who had a positive pre-treatment SLN.

Interestingly, we found a difference in the interpretation of reports as to presence of chemotherapy response by a specialist breast histopathologist and an oncologist. The oncologist tended to regard any mention of fibrosis, necrosis or inflammation as indicating chemotherapy effect, whereas the histopathologist required a specific statement in the report that chemotherapy effect was present. The oncologist involved was also the chief investigator of the trial, and this may represent a subconscious bias given the time invested in establishing a multi centre clinical trial such as neo-tAnGo. Untreated breast cancers can show features such as fibrosis, inflammation and necrosis. In a central review of breast cancers in the tAnGo trial, a multicentre clinical trial looking at the addition of gemcitabine in the adjuvant setting, $8 \%$ of cancers had fibrosis, $22 \%$ had necrosis and $44 \%$ had a lymphocytic inflammatory cell infiltrate (EP, unpublished data). One study found no difference between tumours treated or untreated by chemotherapy with regards to elastosis, necrosis, inflammatory infiltrate or infiltration by macrophages, but there was a significant difference in background breast lobules with cellular atypia, hyalinisation and stromal fibrosis more common post-chemotherapy (Aktepe et al, 1996). Hence, oncologists should look for a specific statement within the pathology report regarding the presence or absence of chemotherapy response and it's degree; if there is no comment regarding chemotherapy effect they should ask the reporting pathologist to go back and review the case, rather than interpret features such as necrosis and inflammation as indicative of response.

Within the neo-tAnGo trial, there was also variation between centres in the management of the axilla. SLNB was performed in 154 patients, $39 \%$ pre-treatment and $61 \%$ post-chemotherapy, with 66 patients going on to a post-treatment ALND. This includes three patients who had a negative SLN pre-chemotherapy, and 29 patients who went on to an immediate ALND after post-treatment SLN, the majority of whom didn't have any form of intraoperative nodal assessment. Hence, the vast majority of patients went directly to a post-treatment ALND. There is an increasing body of evidence that SLN is a reliable technique in the neoadjuvant setting with equivalent results to untreated patients, although there is some controversy as to the optimum timing of the procedure and further clinical trials are awaited to address this issue. (Mamounas et al, 2005; Newman et al, 2007; Hunt et al, 2009; Bear, 2010; Shimazu and Noguchi, 2011).

After the presence of chemotherapy response, the main source of disagreement between reviewers was in lymph node numbers. This was often due to miscounting when there were multiple lymph node specimens, either within the one procedure or as separate procedures. The use of proformas with a summary of the total number of lymph nodes examined makes the report easier to interpret, although on occasion internal inconsistencies in nodal numbers were introduced. The other key reason for disagreement was differences in the interpretation of ITC's by both reviewers and reporting pathologists. The significance of ITC's in the neoadjuvant setting is controversial. One study showed worse outcome in patients with residual nodal disease regardless of the size of the deposit, although the measurements used were $<1 \mathrm{~cm}, 1-10 \mathrm{~cm}$ and $>10 \mathrm{~cm}$, which does not allow discrimination between micrometastases and ITC's (Hennessy et al, 2005). Other series show no difference in outcome between patients with axillary pCR and ITC's including ITC's identified by immunohistochemistry alone (Loya et al, 2009; Sakakibara et al, 2009). The 7th edition of the AJCC TNM staging system recommends that these patients should be classified as ypN0(i+) as for untreated disease, but regarded as not having had a pCR (AJCC, 2010).
In summary, this central review of histopathology reports within a multicentre neoadjuvant clinical trial has revealed wide variation in the handling and reporting of post-chemotherapy breast cancer specimens in different centres across the United Kingdom. The presence of chemotherapy response in the breast and lymph nodes are important prognostic factors, and were commented on in $45 \%$ and $30 \%$ of cases, respectively. This highlights the importance of national guidelines for the reporting of neoadjuvant chemotherapy specimens, as well as the need to engage histopathologists as well as oncologists as key clinicians in neoadjuvant clinical trials.

\section{ACKNOWLEDGEMENTS}

We thank all participating clinicians, histopathologists, research nurses and patients. Data Monitoring Committee members are Professor Lucca Gianni, Professor Craig Henderson and Professor Mark Brady. Research support was obtained from Cancer Research UK: Project grant for Neo-tAnGo clinical trial - C57/A4180 2004-9 (HME); Eli Lilly and Company - unrestricted partnership educational grant for additional trial co-ordination support and free gemcitabine (HME); Bristol Myers Squibb - discounted Taxol. HME, and EP receive support from the Cambridge Experimental Cancer Medicine Centre and the NIHR Cambridge Biomedical Research Centre.

\section{REFERENCES}

AJCC (2010) Breast. In Cancer Staging Handbook from the AJCC Cancer Staging Manual, Edge SB, Byrd DR, Compton CC, Fritz AG, Greene FL, Trotti A (eds) 7th edn, pp 419-460. Springer: New York.

Aktepe F, Kapucuoglu N, Pak I (1996) The effects of chemotherapy on breast cancer tissue in locally advanced breast cancer. Histopathology 29: 63-67.

Bear HD (2010) Neoadjuvant chemotherapy for operable breast cancer: individualizing locoregional and systemic therapy. Surg Oncol Clin N Am 19: $607-626$.

Berruti A, Generali D, Kaufmann M, Puztai L, Curigliano G, Aglietta M, Gianni L, Miller WR, Untch M, Sotiriou C, Daidone M, Conte P, Kennedy D, Damia G, Petronini P, Di Cosimo S, Bruzzi P, Dowsett M, Desmedt C, Mansel RE, Olivetti L, Tondini C, Sapino A, Fenaroli P, Tortora G, Thorne H, Bertolini F, Ferrozzi F, Danova M, Tagliabue E, de Azambuja E, Makris A, Tampellini M, Dontu G, Van't Veer L, Harris AL, Fox SB, Dogliotti L, Bottini A (2011) International expert consensus on primary systemic therapy in the management of early breast cancer: highlights of the Fourth Symposium on Primary Systemic Therapy in the Management of Operable Breast Cancer. Cremona, Italy (2010). J Natl Cancer Inst Monogr 2011: $147-151$.

Bonadonna G, Veronesi U, Brambilla C, Ferrari L, Luini A, Greco M, Bartoli C, Coopmans de Yoldi G, Zucali R, Rilke F (1990) Primary chemotherapy to avoid mastectomy in tumors with diameters of three centimeters or more. J Natl Cancer Inst 82: 1539-1545.

Buzdar AU (2007) Preoperative chemotherapy treatment of breast cancer-a review. Cancer 110: 2394-2407.

Carey LA, Metzger R, Dees EC, Collichio F, Sartor CI, Ollila DW, KlauberDeMore N, Halle J, Sawyer L, Moore DT, Graham ML (2005) American Joint Committee on Cancer tumor-node-metastasis stage after neoadjuvant chemotherapy and breast cancer outcome. J Natl Cancer Inst 97: 1137-1142.

Chevallier B, Roche H, Olivier JP, Chollet P, Hurteloup P (1993) Inflammatory breast cancer. Pilot study of intensive induction chemotherapy (FEC-HD) results in a high histologic response rate. Am J Clin Oncol 16: 223-228.

Chollet P, Amat S, Belembaogo E, Cure H, de Latour M, Dauplat J, Le Bouedec G, Mouret-Reynier MA, Ferriere JP, Penault-Llorca F (2003) Is Nottingham prognostic index useful after induction chemotherapy in operable breast cancer? Br J Cancer 89: 1185-1191.

Dawood S, Broglio K, Kau SW, Islam R, Symmans WF, Buchholz TA, McGuire SE, Meric-Bernstam F, Cristofanilli M, Hortobagyi GN, 
Gonzalez-Angulo AM (2008) Prognostic value of initial clinical disease stage after achieving pathological complete response. Oncologist 13: 6-15.

Dawood S, Merajver SD, Viens P, Vermeulen PB, Swain SM, Buchholz TA, Dirix LY, Levine PH, Lucci A, Krishnamurthy S, Robertson FM, Woodward WA, Yang WT, Ueno NT, Cristofanilli M (2011) International expert panel on inflammatory breast cancer: consensus statement for standardized diagnosis and treatment. Ann Oncol 22: 515-523.

Dowsett M, A'Hern R, Salter J, Zabaglo L, Smith IE (2009) Who would have thought a single Ki67 measurement would predict long-term outcome? Breast Cancer Res 11(Suppl 3): S15.

Earl H, Vallier A-L, Hillier L, Fenwick N, Iddawela M, Hughes-Davies L, Provenzano E, McAdam K, Hickish T, Caldas C (2009) Neo-tAnGo: A neoadjuvant randomised phase III trial of epirubicin/cyclophosphamide (EC) and paclitaxel (T) \pm gemcitabine $(G)$ in the treatment of women with high-risk early breast cancer (EBC): First report of the primary endpoint pathological complete response (pCR). J Clin Oncol 27(Suppl 15abstract 522; poster presentation.

Generali D, Symmans WF, Berruti A, Fox SB (2011) Predictive

immunohistochemical biomarkers in the context of neoadjuvant therapy for breast cancer. J Natl Cancer Inst Monogr 2011: 99-102.

Gounaris I, Britton P, Taylor K, Wallis M, Hiller L, Vallier A-L, Provenzano E, Iddawela M, Earl H, Sinnatamby R (2011) Accuracy of uni-dimensional and volumetric measurements in predicting good pathological response to neoadjuvant chemotherapy in breast cancer patients. Breast Cancer Res Treat 127(2): 459-469.

Hennessy BT, Hortobagyi GN, Rouzier R, Kuerer H, Sneige N, Buzdar AU, Kau SW, Fornage B, Sahin A, Broglio K, Singletary SE, Valero V (2005) Outcome after pathologic complete eradication of cytologically proven breast cancer axillary node metastases following primary chemotherapy. J Clin Oncol 23: 9304-9311.

Hunt KK, Yi M, Mittendorf EA, Guerrero C, Babiera GV, Bedrosian I, Hwang RF, Kuerer HM, Ross MI, Meric-Bernstam F (2009) Sentinel lymph node surgery after neoadjuvant chemotherapy is accurate and reduces the need for axillary dissection in breast cancer patients. Ann Surg 250: 558-566.

Ismail A, Azzawi K, Farouhi P, Earl H, Malata CM (2010) Influence of neoadjuvant chemotherapy on outcomes of immediate breast reconstruction: a retrospective comparison. Plast Reconstr Surg 126(1): $1-11$.

Jones RL, Lakhani SR, Ring AE, Ashley S, Walsh G, Smith IE (2006) Pathological complete response and residual DCIS following neoadjuvant chemotherapy for breast carcinoma. Br J Cancer 94: 358-362.

Loya A, Guray M, Hennessy BT, Middleton LP, Buchholz TA, Valero V, Sahin AA (2009) Prognostic significance of occult axillary lymph node metastases after chemotherapy-induced pathologic complete response of cytologically proven axillary lymph node metastases from breast cancer. Cancer 115: 1605-1612.

Mamounas EP, Brown A, Anderson S, Smith R, Julian T, Miller B, Bear HD, Caldwell CB, Walker AP, Mikkelson WM, Stauffer JS, Robidoux A, Theoret H, Soran A, Fisher B, Wickerham DL, Wolmark N (2005) Sentinel node biopsy after neoadjuvant chemotherapy in breast cancer: results from National Surgical Adjuvant Breast and Bowel Project Protocol B-27. J Clin Oncol 23: 2694-2702.

Marchio C, Sapino A (2011) The pathologic complete response open question in primary therapy. J Natl Cancer Inst Monogr 2011: 86-90.

Mazouni C, Peintinger F, Wan-Kau S, Andre F, Gonzalez-Angulo AM, Symmans WF, Meric-Bernstam F, Valero V, Hortobagyi GN, Pusztai L (2007) Residual ductal carcinoma in situ in patients with complete eradication of invasive breast cancer after neoadjuvant chemotherapy does not adversely affect patient outcome. J Clin Oncol 25: 2650-2655.

Newman EA, Sabel MS, Nees AV, Schott A, Diehl KM, Cimmino VM, Chang AE, Kleer C, Hayes DF, Newman LA (2007) Sentinel lymph node biopsy performed after neoadjuvant chemotherapy is accurate in patients with documented node-positive breast cancer at presentation. Ann Surg Oncol 14: 2946-2952.

Ogston KN, Miller ID, Payne S, Hutcheon AW, Sarkar TK, Smith I, Schofield A, Heys SD (2003) A new histological grading system to assess response of breast cancers to primary chemotherapy: prognostic significance and survival. Breast 12: 320-327.

Oh JL, Nguyen G, Whitman GJ, Hunt KK, Yu TK, Woodward WA, Tereffe W, Strom EA, Perkins GH, Buchholz TA (2007) Placement of radiopaque clips for tumor localization in patients undergoing neoadjuvant chemotherapy and breast conservation therapy. Cancer 110: 2420-2427.

Papa MZ, Zippel D, Kaufman B, Shimon-Paluch S, Yosepovich A, Oberman B, Sadetzki S (2008) Timing of sentinel lymph node biopsy in patients receiving neoadjuvant chemotherapy for breast cancer. J Surg Oncol 98: 403-406.

Penault-Llorca F, Abrial C, Raoelfils I, Cayre A, Mouret-Reynier MA, Leheurteur M, Durando X, Achard JL, Gimbergues P, Chollet P (2008) Comparison of the prognostic significance of Chevallier and Sataloff s pathologic classifications after neoadjuvant chemotherapy of operable breast cancer. Hum Pathol 39: 1221-1228.

Pinder SE, Provenzano E, Earl H, Ellis IO (2007) Laboratory handling and histology reporting of breast specimens from patients who have received neoadjuvant chemotherapy. Histopathology 50: 409-417.

Rastogi P, Anderson SJ, Bear HD, Geyer CE, Kahlenberg MS, Robidoux A, Margolese RG, Hoehn JL, Vogel VG, Dakhil SR, Tamkus D, King KM, Pajon ER, Wright MJ, Robert J, Paik S, Mamounas EP, Wolmark N (2008) Preoperative chemotherapy: updates of National Surgical Adjuvant Breast and Bowel Project Protocols B-18 and B-27. J Clin Oncol 26: 778-785.

Rouzier R, Extra JM, Klijanienko J, Falcou MC, Asselain B, Vincent-Salomon A, Vielh P, Bourstyn E (2002) Incidence and prognostic significance of complete axillary downstaging after primary chemotherapy in breast cancer patients with $\mathrm{T} 1$ to $\mathrm{T} 3$ tumors and cytologically proven axillary metastatic lymph nodes. J Clin Oncol 20: 1304-1310.

Sahoo S, Lester SC (2009) Pathology of breast carcinomas after neoadjuvant chemotherapy: an overview with recommendations on specimen processing and reporting. Arch Pathol Lab Med 133: 633-642.

Sakakibara M, Nagashima T, Kadowaki M, Onai Y, Fujimori T, Yokomizo J, Suzuki H, Fushimi K, Nakatani Y, Miyazaki M (2009) Clinical significance of axillary microresiduals after neoadjuvant chemotherapy in breast cancer patients with cytologically proven metastases. Ann Surg Oncol 16: 2470-2478.

Sataloff DM, Mason BA, Prestipino AJ, Seinige UL, Lieber CP, Baloch Z (1995) Pathologic response to induction chemotherapy in locally advanced carcinoma of the breast: a determinant of outcome. J Am Coll Surg 180: 297-306.

Shimazu K, Noguchi S (2011) Sentinel lymph node biopsy before versus after neoadjuvant chemotherapy for breast cancer. Surg Today 41: 311-316.

Smith IC, Heys SD, Hutcheon AW, Miller ID, Payne S, Gilbert FJ, Ah-See AK, Eremin O, Walker LG, Sarkar TK, Eggleton SP, Ogston KN (2002) Neoadjuvant chemotherapy in breast cancer: significantly enhanced response with docetaxel. J Clin Oncol 20: 1456-1466.

Symmans WF, Peintinger F, Hatzis C, Rajan R, Kuerer H, Valero V, Assad L, Poniecka A, Hennessy B, Green M, Buzdar AU, Singletary SE, Hortobagyi GN, Pusztai L (2007) Measurement of residual breast cancer burden to predict survival after neoadjuvant chemotherapy. J Clin Oncol 25: 4414-4422.

von Minckwitz G, Kummel S, Vogel P, Hanusch C, Eidtmann H, Hilfrich J, Gerber B, Huober J, Costa SD, Jackisch C, Loibl S, Mehta K, Kaufmann M (2008) Neoadjuvant vinorelbine-capecitabine versus docetaxeldoxorubicin-cyclophosphamide in early nonresponsive breast cancer: phase III randomized GeparTrio trial. J Natl Cancer Inst 100: 542-551.

Wolmark N, Wang J, Mamounas E, Bryant J, Fisher B (2001) Preoperative chemotherapy in patients with operable breast cancer: nine-year results from National Surgical Adjuvant Breast and Bowel Project B-18. J Natl Cancer Inst Monogr 30: 96-102.

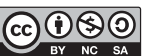

This work is licensed under the Creative Commons Attribution-NonCommercial-Share Alike 3.0 Unported License. To view a copy of this license, visit http://creativecommons. org/licenses/by-nc-sa/3.0/ 